\title{
Pluralisme et éducation
}

Jacques-André Tschoumy

\section{(2) OpenEdition}

Journals

Édition électronique

URL : http://journals.openedition.org/ries/3981

DOI : 10.4000/ries.3981

ISSN : 2261-4265

\section{Éditeur}

Centre international d'études pédagogiques

\section{Édition imprimée}

Date de publication : 7 septembre 1995

Pagination : 137-143

ISSN : 1254-4590

\section{Référence électronique}

Jacques-André Tschoumy, «Pluralisme et éducation », Revue internationale d'éducation de Sèvres [En ligne], 07 | 1995, mis en ligne le 03 octobre 2014, consulté le 21 avril 2019. URL : http:// journals.openedition.org/ries/3981; DOI : 10.4000/ries.3981

Ce document a été généré automatiquement le 21 avril 2019

(c) Tous droits réservés 


\title{
Pluralisme et éducation
}

\author{
Jacques-André Tschoumy
}

«Ce n'est que lorsqu'on a compris qu'aucun homme n'est tout à fait noir comme un diable, ni tout à fait blanc comme un ange, mais que nous sommes tous striés comme des zèbres, et gris comme des ânes, et que l'on en tire les conséquences pratiques, que l'on a véritablement

la possibilité de comprendre son prochain »

(Joseph Kunkel)

« Là où j'entre est toujours aussi là d'où je sors »

(Bernard Comment, jeune écrivain suisse)

\section{Des systèmes trop fermés}

\section{Quel sale temps, ne trouvez-vous pas?}

1 Sale temps pour tous les systèmes fermés: dans les universités américaines, Noirs, Asiatiques, Hispaniques, homosexuels souhaitent désormais vivre dans des foyers séparés. Sale temps pour l'Europe, traversée par un inquiétant courant de purification en Bosnie, en Italie.

2 Sale temps pour la Suisse, pourtant construite sur le pluralisme, et qui se révèle en difficulté à devoir signer la Charte des langues régionales ou minoritaires du Conseil de l'Europe, et qui n'arrive pas, depuis huit ans, à faire accepter à la fois liberté et territorialité dans son projet d'article constitutionnel sur les langues. « Les Romanches ne sont pas des Comanches ", affirmait haut l'ancien président de la Ligue romanche. Pour la première fois, on avait entrepris de réfléchir à une politique des langues propres à façonner la Suisse plurilingue de demain. Une commission d'experts et le Conseil fédéral s'étaient mis en tête d'adapter le droit constitutionnel suisse pour protéger les groupes linguistiques menacés, les Romanches dans le canton des Grisons, et pour donner une 
portée plus dynamique au principe de territorialité, insuffisant dans de nombreuses situations aujourd'hui. La réponse des États cantonaux fut cinglante. Le transfert à niveau fédéral de tels types de problèmes ne se pose pas. L'aire cantonale y suffira. «À clair bornage, bon voisinage", dit l'adage. "Clers comfins fans burs vaschins». Ainsi la territorialité restera principe, alors qu'il n'assure pas la paix des langues, lorsque le rapport entre la majorité et la minorité devient incertain. Ce principe est défensif, figé, fixateur d'immobilisme. Pourra-t-il résister à la poussée interne et externe aux systèmes politiques? Sale temps pour les cantons et les communes aussi! Deux logiques s'affrontent: la territorialité impose la langue d'enseignement et le droit des minorités revendique le droit à une autre langue d'enseignement. Même le Tribunal fédéral a dû se déclarer incompétent sur ce thème. C'est dire que la Suisse plurilingue met son avenir dans des espaces unitaires clos.

3 Sale temps pour les espaces linguistiques monolingues : au dernier recensement fédéral, montent en nombre les alloglottes, passés de $6 \%$ en 1980 à $9 \%$ en 1990 en Suisse. Savezvous que l'italien s'impose peu à peu comme langue intermédiaire, langue d'échanges, entre Grecs et Portugais, par exemple? Or, on ne fait rien de cela, on ne fait rien des registres, des compétences, ni des types d'usage. $37 \%$ de la conversation courante s'exerce sur deux langues au moins au lieu de travail. C'est considérable.

4 Sale temps pour les États dits homogènes: en Europe, les États plurilingues sont désormais les plus nombreux. Et aux États-Unis, l'ascendance du pluralisme est réelle. Sale temps pour les enseignements dits homogènes: le nombre d'étrangers en classes suisses est de $17 \%$, oscillant entre $2 \%$ et $40 \%$. Ce courant est montant. Aux États-Unis, il passera de $24 \%$ en 1992 à $30 \%$ en 2000 et à $50 \%$ en 2050.

5 Sale temps pour quiconque veut mettre son avenir en terre. On ne peut plus penser ainsi aujourd'hui. Il faut penser autrement. Une autre donne s'impose désormais qui ne cherche plus des solutions simples "tout territoire» ou "toute identité " pour des situations complexes. Cette donne ne pourra pas ignorer le pluralisme. Une analyse plus fine s'impose dans cette Europe de changements.

Nous sommes à un tournant. Tous nos systèmes sont à un tournant. Comment allons-nous vivre ensemble ? C'est la seule question qui se pose! Comment vais-je gérer la diversité ? Comment vais-je gérer le pluralisme? Comment intégrer la diversité dans mes fuseaux de pensées, dans mes projets, dans mes mises en œuvre? En d'autres termes, sommes-nous en mesure de nous doter des moyens de gérer le pluralisme autrement qu'en l'excluant, autrement qu'en l'additionnant, qu'en le "mosaïquant »? En regard du modèle « mono » qui a triomphé deux siècles, vais-je accréditer valeur au modèle " pluri » puis au modèle « inter »? Vais-je conférer au pluralisme et à sa gestion valeur de paradigme? Valeur de paradigme à la fois nouveau et nécessaire? Valeur de nouveau cadre conceptuel de nos sociétés postmodernes? Au carrefour de valeurs nouvelles, sommes-nous en mesure d'accréditer valeur à la valeur du pluriel ? Et comment? Voici quatre pistes pour demain.

\section{Une valeur nouvelle : la diversité}

\section{Vers une Europe d'espaces partagés}

7 À l'heure actuelle, le problème se pose en termes de conduite automobile. Comment vaisje tenir le volant d'une voiture dont les appareils de combustion et de transmission sont en train de changer? Il convient aujourd'hui d'ouvrir les yeux sur cette nouvelle Europe 
des chevauchements, des enchevêtrements, de multiples loyautés, de multiappartenances, de « penser » à plusieurs échelles, d'Euro-Régions.

8 L'Europe s'était construite sur ses États. Elle s'est effondrée sur ses États, à Versailles, à Auschwitz, à Treblinka, à Yalta, à Berlin, à Sarajevo, à Szebrenica, à Goradze, à Bihac. Il convient aujourd'hui de penser en espaces partagés nouveaux qui combinent les avantages de l'affirmation territoriale aux avantages de l'affirmation particulariste. Car soyons précis :

- l'affirmation territoriale n'est pas illégitime. Elle assure la paix, offre la moindre forme des tensions internes, exerce un effet-butoir sur les nouveaux arrivants, représente l'outil de communication intercommunautaire à l'intérieur du territoire, organise un «vivre ensemble», relève d'une grande tradition historique venue du Moyen-Age, tient sa légitimité d'en-haut. Mais l'affirmation territoriale repose sur un principe désagréable de purification, donne l'illusion de l'homogène, impose une massification intolérante, cadenasse une uniformité incompatible avec les revendications identitaires. En résumé, l'affirmation territorialiste est légitime, utile, nécessaire même. Mais elle recèle les germes d'abus qu'il convient de juguler.

- l'affirmation particulariste (identitaire, locale, culturelle, migrante, éthique, etc.), de son côté, n'est pas illégitime. Elle relève d'une culture du «vouloir être différent », d'une "échelle du petit». Sa légitimité vient d'en bas. Elle affirme une Europe de valeurs et d'histoire, elle est portée en haut de la vague par tous les points d'Europe, elle se renforce de courants migratoires montants, au point que nombreux sont ceux d'ici qui viennent d'ailleurs. Mais l'affirmation particulariste peut se révéler pernicieuse si l'on n'y prend garde. Elle multiplie les marquages (Beurs, quatrième âge, femmes), aligne les îlots de différences, focalise sur la singularité, enferme dans la particularité, voue un culte à l'aspect distinctif, juxtapose (socialement et culturellement), recherche exclusivement l'aspect particulier, conduit à un inventaire de particularismes, implante numériquement des catégories, contribue à construire une mosaïque sociale, conduit au polycentrisme, à l'éclatement, à l'absence d'interrelation, d'intercommunication, de butoir, de signe rassembleur, et même à l'exclusion réciproque. L'affirmation particulariste, seule, conduit au champ de morts au Rwanda et en ex-Yougoslavie.

\section{Vers une Europe de minorités reconnues}

9 La France a peur de ses Corses, l'Angleterre de ses Écossais, l'Espagne de ses Catalans, la Suisse de ses Jurassiens, l'Italie de ses Ligues.

10 Avant le $\mathrm{XIX}^{\mathrm{e}}$ siècle, on ne se posait pas de problèmes dits de minorités. Or «la démocratie, ce n'est pas la loi de la majorité, c'est la protection de la minorité »; « on ne peut juger des sociétés qu'à la manière dont elles traitent leurs minorités »; «le problème des Noirs n'est pas un problème de Noirs, c'est le problème des Blancs » : ainsi s'exprimaient Albert Camus, le Mahatma Gandhi et Martin Luther King.

11 Or, bonne nouvelle, le droit des minorités est un droit montant en Europe. 


\section{Vers une Europe de langues plurielles}

12 Les nouvelles politiques linguistiques en Europe, à l'impulsion du Conseil de l'Europe, ont conduit à cinq résultats spectaculaires, en Europe, en Suisse, dans la plupart des systèmes éducatifs européens :

- les langues vivantes sont devenues compétences scolaires fondamentales, comme le calcul ou la langue maternelle ;

- c'est la fin du «tout-en-anglais»;

- les langues vivantes ne sont plus apprises par quelques-uns, mais par tous les élèves à l'école ;

- les enfants y apprennent trois langues, et, ainsi la notion de «bilingue » cède le pas à " plurilingue »;

- les enfants y apprennent les langues tôt, très tôt, au primaire, et peu à peu au pré-primaire. C'est donc la fin de l'apprentissage tardif des langues vivantes.

\section{Vers une Europe de citoyennetés composées}

La citoyenneté simple organisait la vie quotidienne autrefois. Le XIX ${ }^{e}$ siècle a même exalté le principe en conférant le droit de nation à un simple État. La religion du prince sera la religion du paysan. Et la langue aussi. Ici on parle français, on vend en français, on enseigne en français, on affiche en français, on renseigne en français, on administre en français. La citoyenneté simple convenait. Elle ne convient plus.

La nouvelle modernité impose pour demain une citoyenneté composée, composée d'identités plurielles marquées de croisements: on parle d'espaces et non plus de territoires. Mon espace sera valaisan, suisse, souvent. Bien sûr. Mais un Valaisan a d'autres espaces qui ne se confondent plus avec les espaces qui l'habitent. Cette extension des champs de citoyennetés s'exerce sur les droits politiques civils et sociaux. C'est la nouvelle forme des champs de citoyennetés, greffées sur des antennes plus que sur des pieds.

\section{Quatre champs}

Issu de notre complexité, issu de notre diversité, est né un nouveau paradigme dont l'émergence s'affiche en espaces partagés, en minorités reconnues, en langues plurielles, en citoyennetés composées. Quatre champs pour une valeur montante de tous nos systèmes. Quatre domaines pour l'ascendance d'une éthique nouvelle, construite sur le pluralisme. Il convient maintenant d'analyser l'impact de ce nouveau cadre conceptuel sur l'éducation en ce domaine exemplaire qu'est la migration du concept interculturel au cours des vingt dernières années.

\section{L'école montre la direction}

16 En regard de cette ascension du pluralisme en tant que composante de nos sociétés postmodernistes, l'école ne reste pas inerte. Spectaculaire aura été sa volonté d'adaptation aux systèmes nouveaux. Mieux, elle montre la direction, ce qui lui arrive 
bien rarement, exceptionnellement même, dans la mesure où son juste rôle est d'être légèrement en retard sur les modes et les évolutions des mentalités.

Issu en 1960 d'une situation d'altérités ignorées, le paradigme « interculturel » a migré. Il s'est dirigé dans un premier temps vers le paradigme d'identités juxtaposées, d'identitésmosaïques : c'était en 1980 ; puis vers le paradigme d'identités articulées à partir de 1990. Cette migration est une évolution déterminante des théories et des pratiques actuelles, de telle sorte que les démarcations sont devenues seuils, puis opérateurs de nuances.

Le concept stigmatise d'abord, collectionne ensuite, nuance enfin; exalte le même d'abord, le pluriel ensuite, le composé enfin; sous-tend l'assimilation, la juxtaposition, puis la transaction; vise l'exclusion, l'alignement, puis la négociation; repose sur l'homogène, la mosaïque, puis le "vivre ensemble »; appréhende la reconnaissance de la différence pour la traiter, l'additionner, puis la négocier ; a pour principe la domination, le foisonnement, l'articulation; précipite l'effacement, l'alignement, puis la gestion de la diversité ; couple identités et similarités d'abord, identités et hétérogénéités ensuite, identités et altérités enfin; a pour paradigme le chien berger d'abord, le catalogue de mesures multi-, inter-, compensatrices et compréhensives ensuite, la hiérarchisation des droits enfin ; vise la négation d'abord, la médication ensuite, l'acculturation enfin ; relève d'une monoculture, d'une multiculture, d'une pluriculturel; affirme l'identité monoculturelle, puis multiculturelle, interculturelle enfin ; projette un modèle « mono ", « multi », « inter »; procède selon un mode soustractif, additif et de trait d'union; repose sur un schéma de dominance, de mosaïque, puis d'articulation; se fait l'agent d'une altérité gommée, reconnue, puis articulée; choisit de voir la différence pour l'effacer d'abord, pour l'ajouter ensuite, pour la valoriser ensuite; charge le regard de différence (s) d'abord, de différence(s) toujours, puis de diversité; s'avère un broyeur, un démultiplicateur, puis un opérateur de nuances; opère au travers d'un monocle, puis d'un binocle, enfin de Varilux.

Nos systèmes sont tous traversés par ces trois courants récents et l'éducation comparée trouve là une grille d'analyse prometteuse. Il est des pays, tels la France, où la langue d'instruction et le système éducatif fondent l'État. Il en est d'autres où le choix des langues d'instruction s'interprète à l'articulation entre territoire et identité. Au Québec, par exemple. Si l'on analyse le système québécois, par carottages, on y trouvera des couches, des strates pédagogiques relevant de ces trois courants en scénarios toujours croisés. L'identité y est encore niée par l'immersion linguistique, restée réservée aux seuls anglophones et confirmée en 1993 aux seuls anglophones,... pour en faire de bons Québécois. Et les cultures autochtones (Inuit) ne sont pas enseignées. À cette couche d'exclusion se superpose la strate de la reconnaissance, avec la reconnaissance des Inuits (six mille Esquimaux) et des Amérindiens (cinquante-quatre mille habitants de communautés autochtones), et leur obtention de droits particuliers. À cette cohabitation, à cette mosaïque, se surajoute un troisième modèle, celui de la transaction interculturelle, de la négociation pluriculturelle, de la valorisation réciproque des cultures, dans un univers jamais neutre, jamais angélique. Dans certains collèges ${ }^{1}$ sont élus un responsable par ethnie, un Comité représentatif, un Comité de liaison intercommunautaire. 


\section{L'école se singularise}

20

21

Comment allons-nous donc traiter la diversité, puisqu'il faut bien vivre ensemble ? À cette question, nous avons tenté de proposer quatre directions de macro-sociétés et un développement de nos systèmes éducatifs. Car il convient d'être et de rester lucide. L'école traditionnelle a toujours été en retard sur l'évolution sociale. Ce retard est normal, l'école ne peut pas s'enflammer pour chaque mode.

Il n'empêche qu'ici, exceptionnellement, l'école est en tête; elle montre la direction, elle intègre tôt un courant nouveau, elle se singularise, elle est pionnière, elle est dans la position du coureur cycliste échappé, qui oscille entre le maintien de son avance sur ses poursuivants et le désir d'économiser ses forces en favorisant son regroupement au peloton de poursuivants. Espace plurilingue, l'école est bien seule dans nos sociétés et nos États dont le discours est bien davantage marqué de contrôle que d'insertion. Même si des communes et des cantons ont mis sur pied, dans les années soixante-dix et quatre-vingt, des structures pour recueillir les avis des communautés migrantes, les structures mises en place pour l'intégration des migrants dépendent toujours des départements de justice et police - aux niveaux national et cantonal. C'est, on en conviendra, une perspective qui ne facilite pas l'intégration, mais qui, au contraire, identifie symboliquement et pratiquement les migrants comme un groupe qu'il faut d'abord contrôler. Il y a décalage.

2 Un instrument didactique de première qualité, Odyssea ${ }^{2}$, vient d'être mis à disposition de toutes les écoles suisses francophones. Au modèle " un peuple, une langue, une nation ", Odyssea propose des scénarios d'ouverture. Ce document cherche à offrir à l'enseignant des clés de compréhension de la modification de sa profession sous l'effet de l'évolution de la population scolaire. Comment faire face aux difficultés provoquées par l'accueil d'un élève venu de l'extérieur ? Pourquoi l'enfant arrive-t-il dans cette classe ? D'où vient-il ? Comment sa famille et lui sont-ils accueillis en Suisse ? L'approche interculturelle induite par cette démarche prend en compte le pluralisme vers lequel tend l'environnement de l'école, tout en recherchant les points unificateurs communs.

Mais un changement de paradigme vers l'ouverture et vers le pluralisme est-il un geste qui puisse s'exercer sur le seul champ scolaire encore longtemps? Les gestes d'ouverture mènent à l'ouverture, mais ont besoin de gestes d'ouverture en retour. Notre propos, ici, est simplement d'éviter la naïveté. Le nouveau paradigme n'est pas neutre. Le « ainsi soitil » n'est jamais suffisant. Si l'on ne veut pas verser dans l'angélisme, s'impose la lucidité : c'est le sens de notre propos.

L'école est devenue espace pluriculturel. Un nouveau paradigme est né à l'école, celui de la gestion de la diversité. Il convient de conforter ce paradigme en termes sociétaux aussi. Car le thème est scolaire et sociétal à la fois. 


\section{NOTES}

1. Collège Drummond à Montréal, par exemple.

2. Toutes les informations sur ce document, qui comprend également un recensement d'outils et moyens didactiques appropriés à ce domaine, peuvent être obtenus auprès de l'IRDP (Institut romand de recherches et de documentation pédagogiques), Service des moyens d'enseignement, faubourg de l'Hôpital 43, case postale 54, 2007 Neuchâtel 7, Suisse

\section{INDEX}

Index géographique : Suisse, France, Europe

Mots-clés : langue de minorité, pluralisme culturel, diversité linguistique, communauté linguistique

\section{AUTEUR}

\section{JACQUES-ANDRÉ TSCHOUMY}

Directeur, Institut romand de recherches et de documentation pédagogique, Neuchâtel, Suisse fr 